\title{
Contribución al conocimiento de la flora alóctona de Galicia (NO Península Ibérica, España)
}

\author{
Xosé Ignacio González-Martínez ${ }^{1}$
}

\begin{abstract}
Resumen: González-Martínez, I. X. 2015. Contribución al conocimiento de la flora alóctona de Galicia (NO Península Ibérica, España). Bot. Complut. 39: 79-85.

Se presenta información sobre 12 taxones de flora vascular exótica gallega, todos ellos herborizados en áreas costeras del término municipal de Ribeira, al SO de la provincia de A Coruña. En este grupo se incluyen 8 novedades regionales (Aeonium haworthii, Aloe mitriformis, Brugmansia $\times$ candida, Nephrolepis cordifolia, Osteospermum ecklonis, Pelargonium capitatum, Sedum mexicanum, Sparaxis tricolor) y 2 provinciales, así como información sobre dos taxones poco conocidos en Galicia. Todos los pliegos testigo se hallan depositados en el Herbario SANT.

Palabras clave: plantas vasculares, flora alóctona, Ribeira, A Coruña.

Abstract: González-Martínez, I. X. 2015. Contribution to the knowlegde of Galician xenoflora (NW Iberian Peninsula, Spain). Bot. Complut. 39: 79-85.

Information on 12 exotic plants of diverse interest for the Galician flora are presented. All of them were collected in Ribeira council (SW of the A Coruña province). The total includes 8 novelties at a regional level (Aeonium haworthii, Aloe mitriformis, Brugmansia $\times$ candida, Nephrolepis cordifolia, Osteospermum ecklonis, Pelargonium capitatum, Sedum mexicanum, Sparaxis tricolor), and 2 provincial novelties. In addition, information on two taxa hardly mentioned in the literature on Galician vascular flora is also included. All the cited specimens are deposited at the
\end{abstract} SANT Herbarium.

Keywords: vascular plants, alien species, Ribeira, A Coruña.

\section{INTRODUCCION}

Como continuación al reciente trabajo de GonzálezMartínez (2014), y con el fin de actualizar en lo posible el conocimiento sobre flora exótica en Galicia, se presentan aquí un total de 12 taxones recolectados en el SO de la provincia de A Coruña, en ambientes litorales o próximos a éstos. Algunos son novedad para Galicia o para la provincia, y además se aporta información sobre dos especies muy poco documentadas en el territorio. Buena parte de las recolecciones fueron realizadas en territorio del «Parque Natural del Complejo Dunar de Corrubedo y Lagunas de Carregal y Vixán» (en adelante, «Parque Natural de Corrubedo»). Esto ha sido debido al trabajo de catalogación de flora vascular que actualmente se está llevando a cabo en dicho espacio. Se citan en cada caso las coordenadas UTM del punto de recolección $[X, Y(m)$, 29T ED50], altitud (m.s.n.m.), autoría y número del pliego del Herbario SANT asignado a los testigos. Además, se hacen diversos comentarios sobre su corología, ecología 0 características morfológicas. Con respecto a la no- menclatura taxonómica, se ha seguido a Flora iberica para las familias publicadas, y a Flora Europaea u otras fuentes actualizadas para el resto. La autoría de los taxones citados en el texto está de acuerdo con las propuestas por Brummitt \& Powell (1992) en sus sucesivas ediciones.

\section{RESULTADOS}

Aeonium haworthii Salm-Dyck ex Webb \& Berthel.

Sempervivum haworthii SD

C: Ribeira, Castiñeiras, Os Areeiros, junto al arenal de O Castro, UTM: 499739, 4708845, 7 m, naturalizada sobre dunas grises (Iberidetum procumbentis) ruderalizadas, 21-III-2014, González-Martínez, SANT 69726; ibidem, Ribeira, Aguiño, cerca del campo de fútbol de A Tasca, UTM: 499054, 4708205, c. 3 m, naturalizada sobre dunas grises (Iberidetum procumbentis), donde coloniza superficies de gran tamaño en compañía del congénere A. arboreum, 02-V-2014, González-Martínez, SANT 69776; ibidem, Ribeira, Corrubedo, cerca de la playa de A Ladeira, UTM: 494862, 4714106, c. 5 m, naturalizada sobre dunas grises (Iberidetum procumbentis) alteradas, 02-V-2014, González-Martinez, SANT 69775.

\footnotetext{
${ }^{1}$ Avda. Miguel Rodríguez Bautista, 23. 15960, Ribeira (A Coruña).xoseignaciogonzalez@yahoo.es Recibido: 14 octubre 2014. Aceptado: 17 noviembre 2014.
} 
Se trata de un Aeonium originario de la parte septentrional de la isla de Tenerife, perteneciente a la sección Leuconium A. Berger, integrada por especies arbustivas ramificadas con flores blanquecinas. Se utiliza como ornamental especialmente en localidades del litoral peninsular.

En la Península Ibérica aparece indicado para Portugal (Domingues de Almeida \& Freitas 2006: 119) y la Comunidad Valenciana (Guillot 2003: 15, Guillot et al. 2009a: 17, Laguna et al. 2014b: 142). En la Isla de Ons (Pontevedra) se ha catalogado el congénere $A$. decorum Webb ex C. Bolle (Bernárdez Villegas 2006: 35), también sobre dunas, pero la fotografía incluida en el trabajo parece corresponder en realidad a la especie que aquí nos ocupa. Ambas son parecidas, pero A. haworthii se distingue por tener tallos grises 0 acastañados, con líneas netamente marcadas, hojas más anchas $(1,5-3 \mathrm{~cm})$ y redondeadas, de ápice cuspidado pero no acuminado, y que a menudo están recubiertas de una pruinescencia glauca (Cullen et al. 2001: 91, 93; Bañares 2008: 479).

Aunque se trata de una planta de crecimiento lento, en alguna de las localidades donde está presente llega a formar agrupaciones de gran tamaño, de hasta $1 \mathrm{~m}$ de altura y c. $4 \mathrm{~m}$ de diámetro, afectando a otras especies autóctonas propias de ecosistemas dunares.

Los presentes testimonios constituyen las primeras referencias en firme para Galicia.

\section{Aloe arborescens Mill.}

C: Ribeira, Carreira, Paralonga, UTM: 499042, 4710988, 51 m, naturalizada en herbazal nitrófilo sobre suelo húmedo (Coleostepho- $G a-$ lactitetum tomentosae), en una zona rocosa de monte bajo con antiguos escombros, 04-V-2014, González-Martínez, SANT 69778.

Utilizada como ornamental en localidades litorales de clima suave, puede escaparse de cultivo e instalarse en ambientes alterados de diversa naturaleza (Güemes 2013: 312). De Galicia ha sido indicada con anterioridad para la provincia de Pontevedra (Güemes op. cit.). En este caso se trata de una pequeña población aislada e integrada por varios ejemplares de gran tamaño, presentes en la zona desde hace al menos 5 años.

Novedad para la provincia de A Coruña.

\author{
Aloe maculata All. \\ A. saponaria (Aiton) Haw. \\ C: Ribeira, Aguiño, UTM: 499069, 4708247, c. 4 m, naturalizada so- \\ bre dunas grises (Iberidetum procumbentis), 02-V-2014, González- \\ Martinez, SANT 69777.
}

Segunda localidad provincial para esta liliácea. Se trata de una especie capense que suele aparecer escapada de cultivo ornamental cerca de asentamientos humanos o zonas muy transitadas (Herrera \& Campos 2010: 33, 39). Para Galicia encontramos la mención de Lence et al. (2005: 5), que la citan de Louro (A Coruña). Sin embargo, dicha referencia no aparece recogida ni en Flora iberica (Güemes 2013: 314) ni en el Catálogo da Flora de Galicia (Romero-Buján 2008), aunque sí figura en la base de datos ANTHOS [08-X-2014]. En la presente localidad aparece repartida en varios puntos, a veces en compañía de las también xenófitas Aeonium haworthii Salm-Dyck ex Webb \& Berthel. y A. arboreum (L.) Webb. \& Berthel. También la hemos encontrado subespontánea en territorio del Parque Natural de Corrubedo (Ribeira, Carreira, A Graña, UTM: 498035, 4709675, $27 \mathrm{~m}$ ), en un herbazal subnitrófilo sobre suelo arenoso, junto a Carpobrotus acinaciformis (L.) L. Bolus.

\section{Aloe mitriformis Mill.}

A. nobilis Haw.- A. perfoliata L.

C: Ribeira, Carreira, A Graña, Parque Natural de Corrubedo, UTM: 497396, 4709763, 15 m, subespontánea en pastizal graminoide de trasduna, junto a las también xenófitas Agave americana y Yucca gloriosa, 26-IX-2014, González-Martínez, SANT 69988.

Para la Península Ibérica encontramos las menciones de Guillot \& Van Der Meer (2005: 7), Guillot et al. (2009b: $33)$ y Güemes (2013: 310). Se trata de una especie próxima a A. maculata All., de la que se diferencia por presentar rosetas con hojas de erectas a erecto-ascendentes, carecer de manchas blancas en el limbo foliar y por presentar una inflorescencia de tipo racemiforme (Guillot et al. 2009b, Güemes 2013).

La cita es novedad para Galicia. Hasta el momento, en territorio gallego se hallan asilvestrados los siguientes representantes del género: Aloe arborescens Mill., A. maculata All., A. mitriformis Mill. y A. vera (L.) Burm. (Güemes 2013: 312, Lence et al:: 2005: 5).

\section{Brugmansia $\times$ candida Pers.}

Datura arborea auct.- Datura $\times$ candida (Pers.) Pasq.- B. aurea Lagerh. $\times$ B. versicolor Lagerh.

C: Ribeira, Cubeliños, UTM: 500112, 4711373, 46 m, subespontánea en herbazal nitrófilo de un solar, sobre suelo alterado y húmedo, 15V-2014, González-Martinez, SANT 69774. 
Solanácea originaria de América del Sur, cultivada como ornamental y que puede llegar a asilvestrarse en medios ruderales y urbanos (Sanz-Elorza et al. 2004: 326). En la presente localidad la encontramos creciendo de forma escasa y próxima a viviendas, de donde probablemente se haya escapado.

No hemos encontrado referencias sobre esta planta para Galicia, por lo que el presente testimonio es novedad regional.

\section{Chamaesyce canescens (L.) Prokh. subsp. canescens}

C: Ribeira, Aguiño, cerca de la playa de 0 Rebalo, UTM: 498888, 4708296, $5 \mathrm{~m}$, cunetas, aceras y base de tapias, sobre suelo arenoso y pisoteado, 04-IX-2012, González-Martínez, SANT 65816.

Euforbiácea ligada a medios antrópicos, considerada un arqueófito originario de la región irano-turania (Benedí 1997: 288, Domingues de Almeida \& Freitas 2006: 121). En este caso aparece de forma escasa en varios puntos próximos entre sí, a menudo en compañía del congénere neófito Ch. maculata (L.) Small. Con posterioridad a la presente recolección, también la hemos encontrado en aceras del perímetro urbano del ayuntamiento de Ribeira (Cubeliños, UTM: 500155, 4711346, 40 m).

El testimonio principal es novedad para A Coruña, y completa además la secuencia provincial para Galicia.

\section{Conyza bilbaoana J. Rémy \\ C. floribunda Kunth?}

C: Ribeira, Carreira, Parque Natural de Corrubedo, junto a las instalaciones de la cantera que se encuentra al lado de la carretera O Vilar-Artes, UTM: 498289, 4712648, 18 m, comunidad ruderal (Sisymbrietalia officinalis) a orillas de un camino, sobre suelo arenoso y húmedo, 28IX-2014, González-Martinez, SANT 69767; ibidem, Ribeira, Carreira, 0 Vilar, Agra do Vilar, Parque Natural de Corrubedo, al lado de una pista que une la aldea de O Vilar con la de Vixán, UTM: 498609, 4710855, $20 \mathrm{~m}$, prado higrófilo (Molinio-Arrhenatheretea) nitrificado, en compañía de Conyza sumatrensis, 02-IX-2014, González-Martínez, SANT 69766; ibidem, Ribeira, Carreira, Vixán, Parque Natural de Corrubedo, UTM: 498640, 4709923, c. 5 m, herbazal higronitrófilo en una leira semiabandonada, creciendo junto a Conyza sumatrensis, 24-IX-2014, González-Martinez, SANT 69989, 70007; ibidem, Ribeira, Carreira, O Vilar, Parque Natural de Corrubedo, a orillas del río Amendo, UTM: 498464, 4710710, 14 m, prado higrófilo nitrificado, junto a Conyza sumatrensis, 30-IX-2014, González-Martínez, SANT 69991, 70006; ibidem, Ribeira, Olveira, Parque Natural de Corrubedo, en las proximidades del C.E.I.P. de la parroquia de Olveira, UTM: 496281, 4715157, 30 $\mathrm{m}$, ruderal en herbazal nitrófilo (Sisymbrietalia officinalis) a orillas de un camino, sobre suelo arenoso y algo húmedo, creciendo acompañada de Conyza sumatrensis, 01-X-2014, González-Martínez, SANT 69990.
Las plantas recolectadas presentaron las siguientes características: porte robusto, tallo de $c .1,5 \mathrm{~m}$ de altura (hemos visto ejemplares que superaban los $2 \mathrm{~m}$ ), con una cubierta más o menos densa de pelos largos y patentes; inflorescencia en panícula amplia, relativamente densa y por lo general de forma romboidal, con ramas profusamente hirsuto-pelosas; hojas con márgenes antrorso-escábridos, las de la parte inferior del tallo y a veces las medias profundamente pinnatipartidas, con lóbulos oblongos y nervios secundarios bien marcados, normalmente provistas de largos pelos rígidos en los márgenes de la parte proximal, al igual que el nervio medio por el envés; involucro de 3-5 mm de longitud, con brácteas glabrescentes 0 con algunos pelos setáceos dispersos, con flores internas generalmente 5-lobadas (aconsejable observar este carácter en el campo) y flores externas no liguladas o con lígulas de menos de $0,5 \mathrm{~mm}$. Este conjunto de caracteres nos llevan a C. bilbaoana (Stanley 1996: 48, Reynolds 1997: 44-45, Mundell 2001: 64, Verloove 2001: 25, Laínz 2002: 352s).

Siguiendo la clave de la Flora dels Països Catalans (Bolòs \& Vigo 1996), y con el fin de apoyar nuestras determinaciones, llegamos a lo que en dicha obra denominan C. blakei (Cabrera) Cabrera. Sin embargo, a este respecto conviene recordar la reveladora nota de Laínz (2002), en la que este autor expone que tanto la iconografía como la descripción de $C$. blakei presentes en la fundamental obra catalana corresponden en realidad y sin lugar a dudas a C. bilbaoana, basándose para ello en la Flora de la Provincia de Buenos Aires (Cabrera 1963) y en materiales de C. blakei prestados por los Royal Botanic Gardens (K). Al parecer, C. blakei, de presencia incierta en la península (Laínz 2002), muestra una inflorescencia en panícula estrecha y alargada, no amplia y más o menos rómbica, y las hojas muy netamente lineares, al igual que sus segmentos en el caso de las pinnatipartidas de la parte inferior del tallo, siendo por tanto muy distinta a C. bilbaoana (Laínz 2002: 353).

Podría decirse, y coincidiendo con Verloove (op. cit.), que $C$. bilbaoana es una planta intermedia entre las próximas $C$. canadensis (L.) Cronq. (=Erigeron canadensis L.) y C. sumatrensis (Retz.) E. Walker (= C. albida Sprengel), aunque difiere significativamente de éstas. La primera suele ser una planta más grácil y de menor tamaño, laxamente pelosa, muestra siempre hojas caulinares enteras o ligeramente dentadas, incluso las de la parte inferior, capítulos con flósculos mayoritariamente 4-lobados y flores externas netamente liguladas (lígulas de $0,5-1 \mathrm{~mm}$ de longitud); la segunda tiene brácteas involucrales pubescentes y muestra un aspecto general cenizo debido a la elevada densidad 
de pelos que recubren tanto el tallo como las ramas de la panícula (Devesa 1987: 12, Bolòs \& Vigo 1996: 718, Aizpuru et al. 1999: 326, Mundell 2001, García Rollán 2005: 298s, García 2008: 327, Blanca et al. 2011: 1628s). El híbrido entre estas dos especies, Conyza $\times$ rouyana Sennen, tampoco se ajusta a los caracteres anteriormente expuestos para las plantas recolectadas.

Desde el punto de vista corológico, la única mención de esta planta para Galicia es la que aparece en Aedo et al. (2001: 29), que la citan de A Capela (Caaveiro, A Coruña). Dicho testimonio es recogido posteriormente por Romero-Buján (2008: 36) en su Catálogo da Flora de Galicia. En el Herbario SANT no hemos encontrado pliego alguno de esta especie.

En el territorio estudiado, C. bilbaoana se encuentra ampliamente dispersa, con una abundancia semejante al resto de congéneres que supuestamente están más extendidos por toda la franja litoral coruñesa. Algo parecido sucede en otras áreas biogeográficas ibéricas, como es el caso de Cantabria (cf. Durán 2014: 79), donde C. bilbaoana muestra el mismo índice de frecuencia-abundancia que C. sumatrensis. Además, existen numerosas citas repartidas por todo el norte peninsular, donde la especie se encuentra en vías de expansión y llega a ser localmente abundante (Aedo et al. 2001, Verloove \& Sánchez Gullón 2008: 153). Parece que $C$. bilbaoana es el representante del género con mayor potencial invasivo (Mundell 2001: 62, Verloove 2001), y en el suroeste de Francia es probable que se haya convertido en la Conyza más abundante (Dauphin 1995: 77s). De igual modo, en algunas áreas del nordeste peninsular puede considerarse en la actualidad una de las adventicias más comunes (Verloove \& Sánchez Gullón 2008).

Resulta sorprendente, por tanto, no encontrar más referencias gallegas de esta xenófita, quizás por haberse asimilado reiteradamente a otras especies del género, principalmente a $C$. canadensis. En cualquier caso, es evidente que la planta está en el campo.

Los presentes testimonios son el segundo y posteriores para Galicia, donde indudablemente debe ser una planta más extendida de lo que puede dar a pensar la práctica ausencia de registros.

\section{Nephrolepis cordifolia (L.) C. Presl}

Polypodium cordifolium L.- Aspidium cordifolium (L.) Swartz- N. auriculata (L.) Trimen nom. rej.

C: Ribeira, Artes, Parque Natural de Corrubedo, UTM: 498751, 4713746, $30 \mathrm{~m}$, escapada de cultivo y colonizando grietas en muros de piedra (Parietarietum judaicae), González-Martínez, SANT 65752; ibidem, Ribeira, Olveira, Sirves, Parque Natural de Corrubedo, UTM: 497067, 4715038, 10 m, naturalizado en una pequeña formación algo alterada de Salix atrocinerea con Laurus nobilis, sobre suelo perhúmedo y asociado a unas palmeras (Phoenix canariensis) plantadas en el lugar hace años, 28-V-2014, González-Martínez, SANT 69772.

Se trata de un helecho subtropical procedente del E Asia y Oceanía, pero que en su sentido más amplio puede considerarse un elemento pantropical (Pyke 2008: 100). Es una especie ampliamente cultivada como ornamental y que se propaga fácilmente por vía vegetativa (Herrero-Borgoñón 2004: 264), colonizando medios antropizados y comportándose como saxícola o como epífito asociado a diversas especies de árboles, especialmente palmáceas (Simões da Silva \& Domingues de Almeida 2000: 182, Herrero-Borgoñón 2004, Pyke 2008, Jiménez Mejías et al. 2010: 476). En la Península Ibérica ha sido mencionado para Cataluña (Pyke 2008), la Comunidad Valenciana (Herrero-Borgoñón 2004, Segarra 2011: 248) y el Peñón de Gibraltar (Galán de Mera et al. 2000: 117).

$N$. cordifolia es muy similar a su congénere $N$. exaltata (L.) Schott, xenófito con el que puede llegar a confundirse y que también se halla presente en la península (Domingues de Almeida \& Freitas 2006: 125, HerreroBorgoñón et al. 2010: 53, Jiménez Mejías et al. 2001). De éste se diferencia, entre otros caracteres, por presentar tubérculos subterráneos (aunque no siempre) y la base de las escamas del raquis oscura, dándole a éstas una aspecto bicoloreado (Coile 1996: 3, Langeland 2001: 6s). Al parecer, ambas especies se hallan en vías de expansión a nivel mundial (Pyke 2008).

También hemos encontrado este helecho en Vixán (Ribeira, Carreira, Parque Natural de Corrubedo, UTM: 498767, 4710046, $20 \mathrm{~m}$ ), colonizando grietas en la base de un muro de piedra.

Los presentes testimonios constituyen novedad para la flora gallega.

Osteospermum ecklonis (DC.) Norl. s. l.

Dimorphotheca ecklonis DC.

C: Ribeira, Artes, Gude, UTM: 499646, 4713418, 50 m, escapada de cultivo e instalada en herbazales y taludes de cunetas, sobre suelo fresco, 21-IV-2014, González-Martínez, SANT 69743.

Debido al gran número de cultivariedades existentes, hemos preferido tratar aquí a este taxón en sentido amplio. Se trata de una planta muy utilizada como ornamental en 
jardinería, y que a veces puede escaparse y colonizar ambientes más o menos alterados próximos a sus cultivos (Guillot 2003: 15, Laguna et al. 2014a: 107s). En España se conoce bien su asilvestramiento en la Comunidad Valenciana (Guillot op. cit., Herrero-Borgoñón 2009: 22, Sanz-Elorza et al. 2012: 113, Laguna et al. 2014a). Para Galicia no existen referencias, por lo que esta aportación es novedad regional.

Por último, además de la localidad de recolección anteriormente indicada, también la hemos visto asilvestrada en otros tres puntos (Ribeira, Artes, Goda, UTM: 498489, 4713371, 33 m, en cunetas; Ribeira, Artes, As Bouciñas, Parque Natural de Corrubedo, UTM: 498653, 4713933, $20 \mathrm{~m}$, herbazal nitrófilo a orillas de un camino; Boiro, Escarís, UTM: 508082, 4721432, 33 m, en cuneta). Se trata, por tanto, de una planta cuyo asilvestramiento en el litoral térmico coruñés es evidente.

\section{Pelargonium capitatum Ait.}

C: Ribeira, Carreira, Paralonga, UTM: 498995, 4710974, 45 m, asilvestrada en zarzal y en herbazal nitrófilo, sobre suelo arenoso, alterado y fresco, 04-V-2014, González-Martínez, SANT 69713.

Se trata de una planta capense utilizada como ornamental, perteneciente al grupo de los pelargonios de hojas fragantes, y que por lo general, y al igual que otros integrantes del género, suele asilvestrarse por vertidos con restos de jardines. En Sanz-Elorza et al. (2004: 318) aparece como especie naturalizada en las Islas Canarias, indicándose posteriormente para Portugal (Domingues de Almeida \& Freitas 2006: 125). La presente cita es novedad para Galicia.

En base a nuestras propias observaciones de campo y a las consultas bibliográficas realizadas (Lence et al. 2005: 31, González-Martínez 2014: 70), parece que hasta el momento en Galicia se hallan asilvestrados, al menos, los siguientes taxones: P. capitatum, P. grandiflorum (Andr.) Willd. ( $P$. $\times$ domesticum L. H. Bailey), Pelargonium $\times$ hortorum L. H. Bailey, P. peltatum (L.) Aiton y P. vitifolium (L.) L'Hér.

\section{Sedum mexicanum Britt.}

C: Ribeira, Carreira, Vixán, Parque Natural de Corrubedo, UTM: 498641, 4710173, $23 \mathrm{~m}$, herbazal higronitrófilo con restos de escombros, 29-IV-2014, González-Martinez, SANT 69702; ibidem, Ribeira, casco urbano, UTM: 501023, 4711448, 6 m, subespontánea en un mu- ro viejo de piedra (Parietarietum judaicae) junto a Aichryson sp., 10VI-2014, González-Martínez, SANT 69773.

Diáfito de origen mejicano que en ocasiones se escapa de cultivo y coloniza ambientes antropizados (SanzElorza et al. 2004: 314). En la Península Ibérica está mencionada para Asturias (Laínz 1970: 21), Cantabria (Aedo et al. 1987: 449), y recientemente para la Comunidad Valenciana (Roselló et al. 2012: 39s).

Novedad para Galicia.

\section{Sparaxis tricolor (Schneev.) Ker Gawl. s. l.}

Ixia tricolor Schneev.

C: Ribeira, Olveira, UTM: 495156, 4714948, 40 m, subespontánea en prado-juncal (Molinio-Arrhenatheretea) próximo a la carretera Ribeira-Corrubedo, sobre suelo arenoso y perhúmedo, 08-IV-2014, González-Martínez, SANT 69709; ibidem, Ribeira, Carreira, 0 Vilar, Eiteiros Rubios, Parque Natural de Corrubedo, a unos $250 \mathrm{~m}$ del Centro de Recepción de Visitantes, UTM: 498123, 4711599, 32 m, varios ejemplares bajo un pinar de Pinus radiata, sobre suelo arenoso y algo húmedo, 04-IV-2014, González-Martínez, SANT 69708.

Este género de la familia de las iridáceas abarca unas cuantas variedades y numerosos híbridos, por lo que aquí hemos tratado a este taxón en sentido amplio. Se trata de una planta originaria del SO de Sudáfrica, y que en territorio ibérico es conocida como adventicia en Portugal desde finales de 1940 (Domingues de Almeida \& Freitas 2006: 128).

Los ejemplares recolectados mostraron flores con estambres equilaterales y tépalos blancos estriados, provistos de una mancha basal intensamente amarilla y de forma más o menos acorazonada. Según Crespo et al. (2013: 403), estos rasgos llevarían a $S$. tricolor.

Todas las plantas fueron encontradas en ecosistemas naturales relativamente alejados de núcleos de población, y los grupos estuvieron integrados por un escaso número de individuos.

La presente aportación, además de novedad para Galicia, parece que también lo es para para la flora española.

\section{AGRADECIMIENTOS}

Al personal del Herbario SANT, Ramiro Iglesias y David GarcíaSan León, por toda la ayuda prestada, a Javier Amigo por sus acertadas sugerencias acerca del texto de Conyza bilbaoana, a Humberto Lens por sus comentarios sobre Nephrolepis cordifolia, y a Antonio Parada por su compañía en muchas de las jornadas de campo disfrutadas. Finalmente, agradecer a un revisor anónimo las correcciones propuestas al texto inicial. 


\section{BIBLIOGRAFÍA}

Aedo, C.; Herrá, C.; Laínz, M.; Loriente, E. \& Moreno, G. 1987. Contribuciones al conocimiento de la flora montañesa, VI. Anales Jard. Bot. Madrid 44(2): 445-457.

Aedo, C.; Aldasoro, J. J.; , Argüelles, J. M.; Carlón, L.; DíeZ riol, A.; Gómez Casares, G.; González del Valle, J. M.; Guillén Oterino, A.; Laínz, M.; Moreno Moral G.; Patallo, J. \& SÁnchez Pedraja, O. 2001. Contribuciones al conocimiento de la flora cantábrica, V. Bol. Cien. Nat. R.I.D.E.A. 47: 7-52.

Aizpuru, I.; AseginolaZa, C.; Uribe-Echebarría, P. M.; UrruTIA, P. \& Zorrakin, I. 1999. Claves ilustradas de la flora del País Vasco y territorios limitrofes. Servicio Central de Publicaciones del Gobierno Vasco, Vitoria-Gasteiz.

BAÑARES, A. 2008. Taxonomic and nomenclatural notes on Crassulaceae of the Canary Islands, Spain. Willdenowia 38: 475-489.

Benedí, C. 1997. Chamaesyce Gray. En: S. Castroviejo, C. Aedo, C. Benedí, M. Laínz, F. Muñoz Garmendia, G. Nieto Feliner \& J. Paiva (Eds.), Flora iberica 8: 286-297. C.S.I.C., Madrid.

Bernárdez Villegas, G. 2006. Estudio florístico de la Isla de Ons. Parque Nacional Maritimo-Terrestre de las Islas Atlánticas de Galicia. Ed. Organismo Autónomo Parques Nacionales, Ministerio de Medio Ambiente y Medio Rural y Marino, Madrid.

Blanca, G. 2011. Conyza Less. En: G. Blanca, B. Cabezudo, M. Cueto, C. Morales Torres \& C. Salazar (Eds.), Flora vascular de Andalucía oriental, $2^{\text {a }}$ Ed.: 1628-1629. Universidades de Granada, Almería, Jaén y Málaga. Granada.

Bolòs, O. \& Vigo, J. 1996. Flora dels Països Catalans III. Pirolàcies - Compostes. Ed. Barcino, Barcelona.

Brummitt, R. K. \& Powell, C. E. 1992. Authors of plant names. Royal Botanic Gardens Kew, London.

Cabrera, A. L. (Ed.). 1963-1970. Flora de la provincia de Buenos Aires, 1-6. Colección Científica del INTA, 4.

Colle, N. C. 1996. Which Boston fern is it? The exotic Nephrolepis cordifolia (L) Presl, or the native Nephrolepis exaltata (L.) Schott. Botany Circular 32. Florida Department of Agriculture and Consumer Services, Gainesville, Florida.

Crespo, M. B. 2013. Iridaceae. En: S. Castroviejo (Coord.), Flora Iberica 20: 400-405. C.S.I.C., Madrid.

Cullen, J.; Knees, S. G. \& Cubey, H. S. (Eds.). 2011. The European garden flora 3. Resedaceae to Cyrillaceae ( $2^{\mathrm{a}} \mathrm{Ed}$.). Cambridge Univertity Press, Cambridge.

Dauphin, P. 1995. Sur l'expansion de Conyza floribunda en Gironde. Bull. Soc. Linn. Bordeaux 23(2): 77-78.

Devesa, J. A. 1987. Conyza Less. En: B. Valdés, S. Talavera \& E. Fernández-Galiano (Eds.), Flora vascular de Andalucía occidental, 3: 11-12. Ed. Ketres, Barcelona.

Domingues De Almeida, J. \& Freitas, H. 2006. Exotic naturalized flora of continental Portugal - A reassessment. Bot. Complut. 30: 117-130.

Durán, J. A. 2014. Catálogo de la flora vascular de Cantabria. Monografías de Botánica Ibérica, 13. Edita: Jolube Consultor y Editor Botánico, Jaca, Huesca.
Galán de Mera, A.; Cortés, J. E. \& Sánchez García, I. 2000. La vegetación del Peñón de Gibraltar. Acta Bot. Malacitana 25: 107-130.

García Rollán, M. 2005. Atlas clasificatorio de la flora de España peninsular y balear 1 ( $3^{\mathrm{a}}$ Ed. Corr.). Ed. Mundi-Prensa. Ministerio de Agricultura, Pesca y Alimentación, Secretaría General Técnica, Centro de Publicaciones, Madrid.

García, X. R. 2008. Guía das plantas de Galicia. Ed. Xerais, Vigo.

GonZÁLEZ-MartínEZ, X. I. 2014. Nuevos datos sobre xenoflora en el término municipal de Ribeira (A Coruña). Nova Acta Ci. Compostelana (Biol.) 21: 65-76.

GüEMES, J. 2013. Aloe L. En: S. Castroviejo, E. Rico, M. B. Crespo, A. Quintanar, A. Herrero \& C. Aedo (Eds.), Flora iberica 20: 308-314. C.S.I.C., Madrid.

GuILLOT, D. 2003. Apuntes corológicos sobre neófitos de la flora valenciana. Fl. Montib. 23: 13-17.

Guillot, D. \& Van der Meer, P. 2005. Nuevos datos de las familias Agavaceae y Aloaceae en la costa mediterránea de la Península Ibérica. Fl. Montib. 30: 3-8.

Guillot, D.; Laguna, E. \& Rosselló, J. A. 2009a. La familia Crassulaceae en la flora alóctona valenciana. Monografías de la revista Bouteloua, 4. Edita: Jolube Consultor y Editor Ambiental, Jaca, Huesca. Recurso electrónico disponible en: www.floramontiberica.org.

Guillot, D.; Laguna, E. \& Rosselló, J. A. 2009b. La familia Aloaceae en la flora alóctona valenciana. Monografías de la revista Bouteloua, 6. Edita: Jolube Consultor y Editor Ambiental, Jaca, Huesca. Recurso electrónico disponible en: www.floramontiberica.org.

Herrera, M. \& CAmpos, J. A. 2010. Flora alóctona invasora en Bizkaia. Instituto de Estudios Territoriales de Bizkaia, Diputación Foral de Bizkaia.

HerRero-Borgoñón, J. J. 2004. Nuevos datos sobre la pteridoflora alóctona ibérica. Acta Bot. Malacitana 29: 263-265.

HERRERO-BORGOÑón, J. J. 2009. Notas sobre flora alóctona valenciana. Fl. Montib. 43: 19-25.

Herrero-Borgoñón, J. J.; Ibars Almonacil, A. M. \& FabreGAT LLUeCA, C. 2010. Acerca de Asplenium seelosii subsp. glabrum y otros pteridófitos escasos en la Comunidad Valenciana. Fl. Montib. 15: 50-54.

Jiménez Mejías, P.; Escudero, M. \& Martín Bravo, S. 2010. Notas sobre neófitos en el curso urbano del río Guadalquivir en Sevilla. Lagascalia 30: 472-481.

Laguna, E.; Ferrer, P.; Guillot, D. \& Roselló, R. 2014a. Sobre algunas especies capenses asilvestradas en tierras valencianas III. Bouteloua 18: 106-115.

Laguna, E.; Gulllot, D.; Roselló, R.; Gómez, M. A.; Ferrer, P.; Deltoro, V. \& PÉrez, P. 2014b. Nuevas citas de plantas alóctonas suculentas asilvestradas en la Comunidad Valenciana. Bouteloua 18: 141-159.

LANGELAND, K. A. 2001. Natural area weeds: distinguishing native and non-native «Boston Ferns» and «Sword Ferns» (Nephrolepis spp.). I.F.A.S. Publication SS-AGR-22, University of Florida, Gainesville. 
LaínZ, M. 1970. Aportaciones al conocimiento de la flora cántabro-astur, IX. Bol. Inst. Estud. Asturianos, Supl. Ci. 15:3-45.

Laínz, M. 2002. Conyza blakei (Cabrera): Cabrera (Compositae), ¿especie peninsular? Anales Jard. Bot. Madrid 59(2): 352-353.

Lence, C.; Acedo, C.; Alonso, R. \& Llamas, F. 2005. Anexo II. Informe final al estudio de flora y vegetación. Proyecto de recuperación y conservación de la Laguna de Louro ( $\mathrm{Mu}$ ros, A Coruña) (inéd.). Fundación Global Nature, Xunta de Galicia y Concello de Muros.

Mundell, T. 2001. Conyza bilbaoana is on its way to you. Bot. Soc. Brit. Isles News 87: 62-65.

PYKE, S. 2008. Contribución al conocimiento de la flora alóctona catalana. Collect. Bot. 27: 95-104.

Reynolds, S. 1997. Conyza bilbaoana also in Ireland. Bot. Soc. Brit. Isles News 74: 44-46.

Romero-Buján, M. I. 2008. Catálogo da Flora de Galicia. Monografías do IBADER 1. Universidade de Santiago de Compostela, Lugo.

Roselló, R.; Laguna, E. \& Guillot, D. 2012. Sedum mexicanum (Crassulaceae), nueva especie alóctona para la flora valenciana. Bouteloua 9: 39-43.
SAnz ElorZA, M.; Dana, E. \& Sobrino, E. 2004. Atlas de las plantas alóctonas invasoras en España. Dirección General para la Biodiversidad, Ministerio de Medio Ambiente, Madrid.

Sanz-ElorzA, M.; Guillot, D. \& Deltoro, V. 2012. La flora alóctona de la Comunidad Valenciana (España). Bot. Complut. 35: 97-130.

SEGARRA, J. G. 2001. Datos sobre la pteridoflora subespontánea ibérica: Cyrtomium falcatum (Dryopteridaceae) y Nephrolepis cordifolia (Nephrolepidaceae). Acta Bot. Malacitana 26: 247-251.

Simões da Silva, A. \& Domingues de Alme1dA, J. 2000. Nephrolepis exaltata (Nephrolepidaceae), naturalized in Europe. Anales Jard. Bot. Madrid 58(1): 182.

Stanley, P. 1996. Conyza bilbaoana J. Rémy, new to South Hampshire (VC 11) and to Britain. Bot. Soc. Brit. Isles News 73: $47-49$.

Verloove, F. 2001. Conyza bilbaoana J. Rémy, Cotoneaster $\times$ wateren Exell en Erigeron karvinskianus DC., nieuw voor de Belgische flora in Kortrijk. Dumortiera 78: 24-27.

Verloove, F. \& SÁnchez Gullón, E. 2008. New records of interesting xenophytes in the Iberian Peninsula. Acta Bot. Malacitana 33: 147-167. 